\title{
Penrose process in a charged axion-dilaton coupled black hole
}

\author{
Chandrima Ganguly ${ }^{1, a}$, Soumitra SenGupta ${ }^{2, b}$ \\ ${ }^{1}$ Department of Applied Mathematics and Theoretical Physics, University of Cambridge, Cambridge CB3 0WA, UK \\ ${ }^{2}$ Department of Theoretical Physics, Indian Association for the Cultivation of Science, Kolkata 700 032, India
}

Received: 30 January 2016 / Accepted: 3 April 2016 / Published online: 18 April 2016

(C) The Author(s) 2016. This article is published with open access at Springerlink.com

\begin{abstract}
Using the Newman-Janis method to construct the axion-dilaton coupled charged rotating black holes, we show that the energy extraction from such black holes via the Penrose process takes place from the axion/Kalb-Ramond field energy responsible for rendering the angular momentum to the black hole. Determining the explicit form for the Kalb-Ramond field strength, which is argued to be equivalent to spacetime torsion, we demonstrate that at the end of the energy extraction process, the spacetime becomes torsion free with a spherically symmetric non-rotating black hole remnant. In this context, applications to physical phenomena, such as the emission of neutral particles in astrophysical jets, are also discussed. It is seen that the infalling matter gains energy from the rotation of the black hole, or equivalently from the axion field, and that it is ejected as a highly collimated astrophysical jet.
\end{abstract}

\section{Introduction}

The energy extraction process from the black hole following the Penrose process gave rise to a new understanding of black hole mechanics. Thermodynamic principles associated with black hole geometry gained enormous interest in recent times in the context of string theory where different kinds of black holes have been investigated in the light of this principle. Newman and Janis $[1,2]$ showed that different kinds of inequivalent black holes can be obtained from known solutions; for example, from the Schwarzschild black hole, the Kerr solution can be obtained, while the Kerr-Newman metric emerges from the Reissner-Nordstrom geometry using the Newman-Janis prescription.

In an alternative approach in string theory, Sen $[3,4]$ showed that, using T-duality in the low energy string effec-

\footnotetext{
a e-mail:cg528@cam.ac.uk

be-mail: tpssg@iacs.res.in
}

tive action, one can generate inequivalent black hole solutions such as charged black hole solutions from uncharged black hole solutions. After the development of dilaton and axion-dilaton solutions [5-7], it was shown by [8] that the Newman-Janis method can be used to develop the charged axion-dilaton solutions from a pure dilaton coupled solution, where the axion field plays the role of the angular momentum parameter of the black hole.

It is well known in a string inspired scenario that in four space-time dimensions, the massless axions are dual to the third rank field strength of a second rank antisymmetric tensor field (known as Kalb-Ramond field) which appears in the massless sector of closed bosonic string theories. While this KR field can be interpreted as an external gauge field defined on a Riemannian manifold, there is an alternative viewpoint where the third rank antisymmetric field strength corresponding to the second rank KR field can also be identified with space-time torsion [9-13] implying a non-Riemannian geometric structure where the torsion field is completely antisymmetric in all its three indices. Either of these interpretations leads to the same action for the low energy supergravity sector of string theory. It is therefore interesting to study the role of the dilaton (whose vacuum expectation value determines the string coupling) as well as the role of the axion in the energy extraction process from the black hole via the Penrose process.

In this work we try to address this question in the context of string inspired axion-dilaton scalar coupled black hole solution obtained via the Newman-Janis prescription. We shall show that, starting from a space-time endowed with nonvanishing KR-energy density (in other words non-vanishing torsion), the energy extraction process eventually results into a static non-rotating dilaton coupled black hole where, at the end of the energy extraction process, the axion/KR field strength becomes zero, leading to a static Riemannian spacetime.

We then establish a connection between our findings and experimental results regarding the emission of neutral parti- 
cles in astrophysical jets, believed to be from rotating black holes.

\section{Axion-dilaton coupled gravity}

The equations of motion for the Einstein-Maxwell axiondilaton coupled gravity can be obtained from the action,

$$
\begin{aligned}
\mathcal{S}= & -\frac{1}{16 \pi} \int \mathrm{d}^{4} x \sqrt{-g}\left(R-2 \partial_{\mu} \Phi \partial^{\mu} \Phi-\frac{1}{2} e^{4 \Phi} \partial_{\mu} \Theta \partial^{\mu} \Theta\right. \\
& \left.+e^{-2 \Phi} F_{\mu \nu} F^{\mu \nu}+\Theta F_{\mu \nu} \tilde{F}^{\mu \nu}\right),
\end{aligned}
$$

where $R$ is the Ricci scalar with respect to the space-time metric $g_{\mu \nu}$ while $F_{\mu \nu}$ and $\tilde{F_{\mu \nu}}$ are the Maxwell tensor and its dual. Here $\Theta$ and $\Phi$ represent the axion and the dilaton fields, respectively. Such an action has its origin in the low energy string effective action in four dimensions. There have been well-known methods like $O(d) X O(d)$ duality symmetry to generate charged black hole solutions from uncharged black hole solutions $[3,4,14]$. Here we adopt a different solution generating technique, following Newman and Janis to obtain a rotating solution from a non-rotating black hole solution [1,2].

Without trying to obtain the solution from the action directly, we first focus on pure dilaton coupled gravity.

The solution to the pure dilaton coupled gravity has been obtained in [5-7]. It resembles the Reissner-Nordstrom solution with dilaton scalar and is spherically symmetric. This can be written

$$
\begin{aligned}
\mathrm{d} s^{2}= & \left(\frac{1-\frac{r_{1}}{r}}{1+\frac{r_{2}}{r}}\right) \mathrm{d} t^{2}-\left(\frac{1-\frac{r_{1}}{r}}{1+\frac{r_{2}}{r}}\right)^{-1} \mathrm{~d} r^{2}-r^{2}\left(1+\frac{r_{2}}{r}\right) \\
& \times\left(\mathrm{d} \theta^{2}+\sin ^{2} \theta \mathrm{d} \phi^{2}\right) ;
\end{aligned}
$$

$r_{1}$ and $r_{2}$ can be expressed in terms of the mass and charge of the dilaton black hole as

$r_{1}+r_{2}=2 M$

and

$r_{2}=\frac{Q^{2}}{M}$.

The dilaton field $\Phi$ is given by

$e^{2 \Phi}=\frac{1}{1+\frac{r_{2}}{r}}$

where the asymptotic value of the dilaton $\Phi_{0}$ above and in all other computations in this paper is taken to be zero. The electrostatic potential is

$A=\frac{-\frac{Q}{r}}{1+\frac{r_{2}}{r}}$.
In analogy to the work done by Newman and Janis $[1,2]$ in obtaining the axisymmetric rotating black hole Kerr solution from the spherically symmetric Schwarzschild solution, we can now construct the axisymmetric black hole solution from the spherically symmetric solution obtained from dilaton coupled gravity. This algorithm works by first transforming the metric to the outgoing Eddington-Finkelstein coordinates, given by

$\mathrm{d} t=\mathrm{d} u+\left(\frac{1-\frac{r_{1}}{r}}{1+\frac{r_{2}}{r}}\right)^{-1} \mathrm{~d} r$.

The metric in these coordinates then becomes

$\mathrm{d} s^{2}=\left(\frac{1-\frac{r_{1}}{r}}{1+\frac{r_{2}}{r}}\right) \mathrm{d} u^{2}+2 \mathrm{~d} u \mathrm{~d} r-r^{2}\left(1+\frac{r_{2}}{r}\right) \mathrm{d} \Omega^{2}$.

We then express the metric in terms of the null tetrad vectors in the following form:

$g^{\mu \nu}=l^{\mu} n^{\nu}+l^{v} n^{\mu}-m^{\mu} \bar{m}^{\nu}-m^{\nu} \bar{m}^{\mu}$.

The null tetrad vectors which would give back the correct metric are

$$
\begin{aligned}
l^{\mu} & =\delta_{1}^{\mu}, \\
n^{\mu} & =\delta_{0}^{\mu}-\frac{1}{2}\left(\frac{1-\frac{r_{1}}{r}}{1+\frac{r_{2}}{r}}\right) \delta_{1}^{\mu}, \\
m^{\mu} & =\frac{1}{\sqrt{2} r \sqrt{1+\frac{r_{2}}{r}}}\left(\delta_{2}^{\mu}+\frac{i}{\sin \theta} \delta_{3}^{\mu}\right) .
\end{aligned}
$$

We then allow $r$ to take complex values and write the tetrad vectors in the following manner:

$$
\begin{aligned}
l^{\mu} & =\delta_{1}^{\mu}, \\
n^{\mu} & =\delta_{0}^{\mu}-\frac{1}{2}\left(\frac{1-\frac{r_{1}}{2}\left(\frac{1}{r}+\frac{1}{\bar{r}}\right)}{1+\frac{r_{2}}{2}}\left(\frac{1}{r}+\frac{1}{\bar{r}}\right)\right) \delta_{1}^{\mu}, \\
m^{\mu} & =\frac{1}{\sqrt{2} \bar{r} \sqrt{1+\frac{r_{2}}{2}\left(\frac{1}{r}+\frac{1}{\bar{r}}\right)}}\left(\delta_{2}^{\mu}+\frac{i}{\sin \theta} \delta_{3}^{\mu}\right) .
\end{aligned}
$$

The null tetrad thus obtained is then made to undergo the complex transformation which introduces a parameter ' $a$ '. This transformation is such that the resulting metric is a real function of complex arguments $r$ and $u$, which are given as

$r \rightarrow r+i a \cos \theta$,
$u \rightarrow u-i a \cos \theta$,
$\theta \rightarrow \theta, \quad \phi \rightarrow \phi$.

We require that $r^{\prime}$ and $u^{\prime}$ remain real, and we consider the null vectors under this coordinate transformation. They take the following form:

$$
l^{\prime \mu}=\delta_{1}^{\mu} \text {, }
$$




$$
\begin{aligned}
n^{\prime \mu}= & \delta_{0}^{\mu}-\frac{1}{2}\left(\frac{1-\frac{r_{1} r}{\Sigma}}{1+\frac{r_{2} r}{\Sigma}}\right) \delta_{1}^{\mu}, \\
m^{\prime \mu}= & \frac{1}{\sqrt{2}\left(r^{\prime}+i a \cos \theta\right)} \frac{1}{\sqrt{1+\frac{r_{2} r^{\prime}}{\Sigma}}} \\
& \times\left(i a \sin \theta\left(\delta_{0}^{\mu}-\delta_{1}^{\mu}\right) \delta_{2}^{\mu}+\frac{i}{\sin \theta} \delta_{3}^{\mu}\right)
\end{aligned}
$$

where $\Sigma=r^{\prime 2}+a^{2} \cos ^{2} \theta$.

Putting these forms of the null tetrad vectors into the form of the metric using (9), and then transforming back from the Eddington-Finkelstein coordinates, we get

$$
\begin{aligned}
\mathrm{d} s^{2}= & -\left(1-\frac{2 M r}{\tilde{\Sigma}}\right) \mathrm{d} t^{2} \\
& -\frac{4 a M r}{\tilde{\Sigma}} \sin ^{2} \theta \mathrm{d} t \mathrm{~d} \phi-\frac{\tilde{\Sigma}}{\Delta}\left(\mathrm{d} r^{2}+\Delta \mathrm{d} \theta^{2}\right) \\
& +\left\{r\left(r+r_{2}\right)+a^{2}+\frac{2 M r a^{2} \sin ^{2} \theta}{\tilde{\Sigma}}\right\} \sin ^{2} \theta \mathrm{d} \phi^{2}
\end{aligned}
$$

The axion field $\Theta$ is given by

$\Theta=\frac{Q^{2}}{M} \frac{a \cos \theta}{r^{2}+a^{2} \cos ^{2} \theta}$.

Here $r_{2}=\frac{Q^{2}}{M} e^{2 \Phi_{0}}$. Thus we see that with the aid of a coordinate transformation we are able to arrive at a solution with non-vanishing axion field. This field is given by the parameter of the transformation ' $a$ ' and its effect is to render the black hole with non-zero angular momentum and thereby making it a rotating black hole.

The non-vanishing components of the third rank antisymmetric dual Kalb-Ramond (KR) field strength $H_{\mu \nu \alpha}$ can now be determined from the expression of the axion field $\Theta$ using the duality relation which is given by

$H_{a b c}=\frac{1}{2} e^{4 \Phi} \epsilon_{a b c d} \partial^{d} \Theta$.

This is actually a closed string mode. These turn out to be

$$
\begin{aligned}
H_{023}= & \frac{\left\{r\left(r+r_{2}\right)+a^{2} \cos ^{2} \theta-2 M r\right\}\left[\left\{r\left(r+r_{2}\right)+a^{2} \cos ^{2} \theta\right\}\left\{r\left(r+r_{2}\right)+a^{2}\right\}+2 M r a^{2} \sin ^{2} \theta\right]-\left(2 M r a \sin ^{2} \theta\right)^{2}}{\left\{r\left(r+r_{2}\right)+a^{2} \cos ^{2} \theta\right\}^{2}} \\
& \times \frac{2 Q^{2} r a \cos \theta}{M\left(r^{2}+a^{2} \cos ^{2} \theta\right)^{2}}\left[\frac{r\left(r+r_{2}\right)-2 M r+a^{2} \cos ^{2} \theta-2 a M r \sin ^{2} \theta}{r\left(r+r_{2}\right)+a^{2} \cos ^{2} \theta}\right]^{-1} \\
& \times\left[\left(\frac{r\left(r+r_{2}\right)+a^{2} \cos ^{2} \theta}{r\left(r+r_{2}\right)-2 M r+a^{2}}\right) \times\left\{\left(r\left(r+r_{2}\right)+a^{2} \cos ^{2} \theta\right)\left(r\left(r+r_{2}\right)+a^{2}\right)+2 M r a^{2} \sin ^{2} \theta\right\}\right]^{-1}
\end{aligned}
$$

and

$$
\begin{aligned}
H_{031}= & \frac{\left\{r\left(r+r_{2}\right)+a^{2} \cos ^{2} \theta-2 M r\right\}\left[\left\{r\left(r+r_{2}\right)+a^{2} \cos ^{2} \theta\right\}\left\{r\left(r+r_{2}\right)+a^{2}\right\}+2 M r a^{2} \sin ^{2} \theta\right]+\left(2 M r a \sin ^{2} \theta\right)^{2}}{\left\{r\left(r+r_{2}\right)+a^{2} \cos ^{2} \theta\right\}^{2}} \\
& \times \frac{Q^{2}}{M}\left[\frac{a \sin \theta}{r^{2}+a^{2} \cos ^{2} \theta}+\frac{a^{2} \sin 2 \theta \cos \theta}{\left.r^{2}+a^{2} \cos ^{2} \theta\right)^{2}}\right]\left[\frac{r\left(r+r_{2}\right)-2 M r+a^{2} \cos ^{2} \theta-2 a M r \sin ^{2} \theta}{r\left(r+r_{2}\right)+a^{2} \cos ^{2} \theta}\right]^{-1} \\
& \times\left[\left(\frac{r\left(r+r_{2}\right)+a^{2} \cos ^{2} \theta}{r\left(r+r_{2}\right)-2 M r+a^{2}}\right) \times\left\{\left(r\left(r+r_{2}\right)+a^{2} \cos ^{2} \theta\right)\left(r\left(r+r_{2}\right)+a^{2}\right)+2 M r a^{2} \sin ^{2} \theta\right\}\right]^{-1}
\end{aligned}
$$

where $\Delta=r\left(r+r_{2}\right)-2 M r+a^{2}$ and $\tilde{\Sigma}=r\left(r+r_{2}\right)+$ $a^{2} \cos ^{2} \theta$. Comparing with the solution obtained from [4], we have the electromagnetic potential:

$A=-\frac{Q r}{\tilde{\Sigma}}\left(\mathrm{d} t-a^{2} \sin \theta \mathrm{d} \phi\right)$.

The dilaton field $\Phi$ is

$$
e^{2 \Phi}=\frac{r^{2}+a^{2} \cos ^{2} \theta}{r\left(r+r_{2}\right)+a^{2} \cos ^{2} \theta} .
$$

These expressions of the KR field strength determine the energy density of the KR field which acts is the source of the rotational energy of the black hole. It is to be noted that the KR field vanishes when the parameter $a$ vanishes. As the parameter $a$ is a measure of the angular momentum of the black hole, as shall be seen later, it can be concluded that a non-vanishing KR field in this scenario is related to the rotational energy of the black hole.

Thus we arrive at the existence of non-spherically symmetric solutions for the Kalb-Ramond field strength corresponding to this class of rotating black hole solutions where 
the axion and therefore the Kalb-Ramond field acts as the source of rotation of the black hole and thus generates an axisymmetric solution from a spherically symmetric dilaton solution. As discussed earlier, this can also be interpreted as the torsion in the background spacetime and thus from a Riemannian spacetime we generate an Einstein-Cartan-like spacetime.

\section{Horizon structure}

As we have seen in the previous section, the metric obtained from the Einstein-Maxwell axion-dilaton gravity resembles the Kerr rotating black hole metric with a modification caused by the term $r_{2}$. This is actually the asymptotic value of the dilaton along with a multiplicative constant. Thus we have a Kerr solution modified by the presence of the dilaton field. We now study the horizon structure of this metric. 0

We begin with the coordinate singularity arising from $\Delta=$

$r\left(r+r_{2}\right)-2 M r+a^{2}=0$.

The event horizons are then at

$r_{ \pm}=\left(M-\frac{r_{2}}{2}\right) \pm \sqrt{\left(M-\frac{r_{2}}{2}\right)^{2}-a^{2}}$.

The metric shows time translation symmetry as well as axial symmetry, due to the independence of $t$ and $\phi$ in the metric components. This gives rise to a timelike Killing vectors $\tau^{\mu}$ and a Killing vector arising out of the axial symmetry $\eta^{\mu}$. The inner product of the timelike Killing vector gives us,

$\tau_{\mu} \tau^{\mu}=1-\frac{2 M r}{r\left(r+r_{2}\right)+a^{2} \cos ^{2} \theta}$.

This quantity becomes positive at the event horizon and like the case of the Kerr black hole it becomes zero at a hypersurface at a distance greater than the radius of the event horizon. Let this radius be $r_{e}$ and given by

$r_{e}=\left(M-\frac{r_{2}}{2}\right) \pm \sqrt{\left(M-\frac{r_{2}}{2}\right)^{2}-a^{2} \cos ^{2} \theta}$.

The region in between $r_{e}$ and $r_{+}$is known as the ergosphere.

The maximum angular velocity that a particle can travel with, due to the rotation of the black hole, is that of a photon on the event horizon $r_{+}$, moving along an equatorial orbit. We define the angular velocity of this photon to be the angular velocity of the black hole. In this case, as the photon is moving along the event horizon, we have the condition

$\Delta=0$

Therefore we have

$r\left(r+r_{2}\right)+a^{2}=2 M r$
Considering a light ray that is being emitted in the $\phi$ direction in the $\theta=\pi / 2$ equatorial orbit, we have the condition for it to be null:

$\mathrm{d} s^{2}=g_{t t} \mathrm{~d} t^{2}+2 g_{t \phi} \mathrm{d} t \mathrm{~d} \phi+g_{\phi \phi} \mathrm{d} \phi^{2}=0$.

This yields,

$\frac{\mathrm{d} \phi}{\mathrm{d} t}=-\frac{g_{t \phi}}{g_{\phi \phi}} \pm \sqrt{\left(\frac{g_{t \phi}}{g_{\phi \phi}}\right)^{2}-\frac{g_{t t}}{g_{\phi \phi}}}$.

Using this equation, we find the angular velocity of the photon to be

$\frac{\mathrm{d} \phi}{\mathrm{d} t}=0$

and

$\frac{\mathrm{d} \phi}{\mathrm{d} t}=\frac{a}{2 M r_{+}}=\Omega_{H}$

The zero solution indicates that the photon is not moving at all in this frame. The non-zero solution shows the angular velocity with which the photon is being dragged around is in the same direction as the hole's rotation. The angular velocity of the event horizon itself is defined as the maximum angular velocity of a particle at the horizon. This quantity is given by

$\omega_{H}=\frac{a}{2 M r_{+}}$.

\section{Energy extraction and the Penrose process}

A calculation of the conservation of energy and angular momentum for a particle in the ergosphere can lead to an energy extraction process demonstrated by Penrose in the case of a Kerr black hole. Here we consider the procedure followed in [15]. Briefly, this shows that a particle, in the ergosphere breaks up into two parts such that one part falls into the event horizon of the black hole and the other escapes into the external universe, in a manner in which, the escaping particle can be shown to have more energy than the original particle before it breaks apart into two fragments. This excess energy is said to be gained from the rotational energy of the black hole. By repeating this process again and again the black hole slows down gradually until the rotation stops altogether and the Kerr black hole becomes a Schwarzschild black hole.

Estimation of energy extraction Assume that a particle on entering the ergosphere breaks up into two particles $A$ and $B$. Before breaking up, the four momentum of the whole particle was $p^{(0) \mu}$, and the energy was $E=-\tau_{\mu} p^{(0) \mu}$. This energy is positive and conserved along its geodesic. When the particle breaks up into smaller particles, then the four momentum and energy are conserved. 
$p^{(0) \mu}=p^{(A) \mu}+p^{(B) \mu}$,

$E^{(0)}=E^{(A)}+E^{(B)}$.

Here $p^{(A) \mu}$ and $p^{(B) \mu}$ are the four momentums of the two constituent particles and $E^{(A)}$ and $E^{(B)}$ are the corresponding energies. From the above equations the following analysis can be made. If the momentum of the second particle be such that its energy is negative, Penrose showed that the initial momentum can be arranged so that afterwards a geodesic trajectory can be followed from the Killing horizon back into the external universe. Energy still remains conserved along this path and we have

$E^{(A)}>E^{(0)}$.

This implies that the energy with which the first particle leaves the Killing horizon is more than the energy with which it entered. This energy extraction has come from the rotational energy of the black hole, which in effect originates from the energy density of the axion/KR field.

We now define a new Killing vector, taking into account the modification caused by the dilaton field, by

$\chi^{\mu}=\tau^{\mu}+\left(1-\frac{r_{2}}{4 M}\right)^{-1} \Omega_{H} \eta^{\mu}$.

For a particle $B$ which crosses the event horizon moving forward in time

$p^{(B) \mu} \chi_{\mu}<0$.

Using the definitions of $E$ and $L$ as $E=-\tau_{\mu} p^{\mu}$ and $L=$ $\eta_{\mu} p^{\mu}$, we get

$L^{(B)}<\left(1-\frac{r_{2}}{4 M}\right) \frac{E^{(B)}}{\Omega_{H}}$.

As $E^{(B)}$ is taken to be negative and $\Omega_{H}$, the black hole's angular momentum, is positive, the particle must have a negative angular momentum. In other words it must be moving against the hole's rotation. Once the particle $A$ escapes into the external universe and the particle $B$ falls into the event horizon, the energy and angular momentum of the black hole are changed by the negative contributions of the particle $B$ that has fallen into it. Thus we have

$$
\begin{aligned}
& \delta M=E^{(B)}, \\
& \delta J=L^{(B)} .
\end{aligned}
$$

The total angular momentum of the black hole $J$ is given by

$$
J=M a .
$$

Thus Eq. (43) becomes

$\delta J<\left(1-\frac{r_{2}}{4 M}\right) \frac{\delta M}{\Omega_{H}}$.
To find the limit of energy extraction in the case of a Kerr black hole, a quantity known as the irreducible mass is defined as follows:

$M_{\text {irr }}^{2}=\frac{A}{16 \pi}$

where $A$ is the area of the event horizon. $\delta\left(M_{\text {irr }}^{2}\right)$ can be shown to be always greater than zero.

We find the area of the black hole at the event horizon by defining a constant-time hypersurface at $r=r_{+}$. The metric of this hypersurface is

$\mathrm{d} \Lambda^{2}=\tilde{\Sigma}_{+}^{2} \mathrm{~d} \theta^{2}+\left(\frac{2 M r_{+}}{\tilde{\Sigma}}\right)^{2} \sin ^{2} \theta \mathrm{d} \phi^{2}$.

The area is then calculated from the relation

$\mathrm{d} A=\sqrt{g_{\theta \theta} g_{\phi \phi}} \mathrm{d} \theta \mathrm{d} \phi$,

which gives

$A=2 M r_{+} \int_{0}^{\pi} \sin \theta \mathrm{d} \theta \int_{0}^{2 \pi} \mathrm{d} \phi=8 \pi M r_{+}$.

When the Kerr parameter ' $a$ ' goes to zero, we get the area of the event horizon of a charged dilaton black hole

$A_{\text {dilaton }}=16 \pi M\left(M-\frac{r_{2}}{2}\right)$.

The square of the irreducible mass gives us

$M_{\text {irr }}^{2}=\frac{A}{16 \pi}=\frac{M r_{+}}{2}$,

which turns out to be

$M_{\mathrm{irr}}^{2}=\frac{1}{2}\left[\left(M^{2}-\frac{M r_{2}}{2}\right)+\sqrt{\left(M^{2}-\frac{M r_{2}}{2}\right)^{2}-J^{2}}\right]$.

Thus,

$\delta\left(M_{\mathrm{irr}}^{2}\right)=\frac{a}{2 \sqrt{\left(M-\frac{r_{2}}{2}\right)^{2}-a^{2}}}\left[\left(1-\frac{r_{2}}{4 M}\right) \Omega_{H}^{-1} \delta M-\delta J\right]$.

The right hand side is greater than zero by the inequality (33) and thus we can say that $M_{\text {irr }}$ is the limit to which the energy of the black hole can be extracted.

The maximum amount of energy extraction can be defined by taking into consideration the energy of the black hole that remains after the energy of the axion from the KR field has been exhausted. In the absence of the axion, we are left with pure dilaton coupled gravity and the energy that remains is the energy of the charged non-rotating dilaton black hole.

From the entropic definition of the irreducible mass (as the black hole entropy is proportional to the area of its event horizon) we can define a quantity similar to the irreducible 


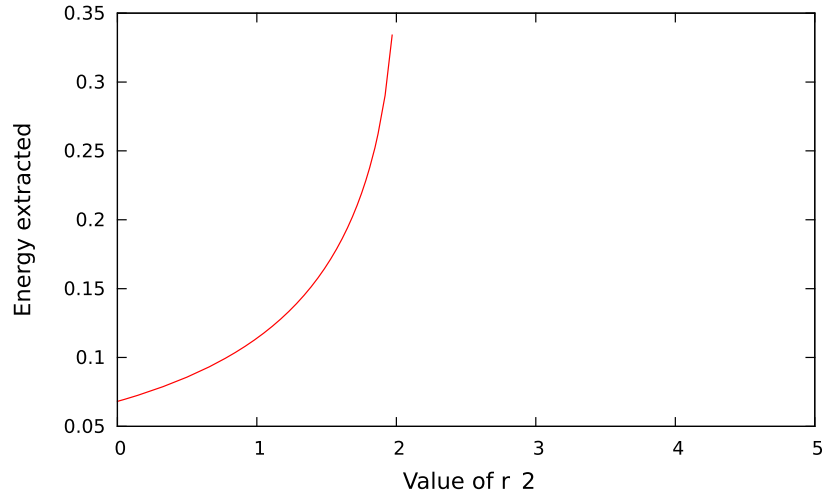

Fig. 1 Variation of energy extraction with dilaton

mass of the charged axion-dilaton black hole. Let the square of this quantity $M_{\text {dilaton }}$ be defined by

$M_{\text {dilaton }}^{2}=\frac{A_{\text {dilaton }}}{16 \pi}=M\left(M-\frac{r_{2}}{2}\right)$.

The maximum amount of energy extracted before the rotation of the black hole stops can now be found as follows:

$\Delta M=M_{\text {dilaton }}-M_{\text {irr }}$.

This quantity becomes

$$
\begin{aligned}
\Delta M & =\sqrt{M\left(M-\frac{r_{2}}{2}\right)} \\
& -\sqrt{\frac{M}{2}\left\{\left(M-\frac{r_{2}}{2}\right)+\sqrt{\left(M-\frac{r_{2}}{2}\right)^{2}-a^{2}}\right\}} .
\end{aligned}
$$

Thus the ratio of the energy extracted to the dilaton mass can be written as

$$
\frac{\Delta M}{M_{\text {dilaton }}}=1-\sqrt{\frac{1}{2}\left\{1+\sqrt{1-a^{2}\left(M-\frac{r_{2}}{2}\right)^{-2}}\right\}} .
$$

Remembering that $r_{2}=\frac{Q^{2}}{M}$ the variation of the energy extraction with the dilaton parameter $r_{2}$ is plotted, taking $M=2$ and $r_{2}=1$, as follows. From the above expression we have $Q^{2}=2$ (see Fig. 1).

As can be seen from the figure the amount of energy extraction as well as the rate of change of energy extracted increases with the increase in the charge $Q$ and decreases with increase in mass $M$ (Fig. 2).

Here too, the value of energy extraction reduces with the decrease in the value of the axion as does the rate of energy extraction with respect to the axion field. When the axion parameter goes to zero, the ergosphere vanishes and the energy extraction stops, as expected.

Astrophysical black holes The Penrose process of energy extraction has been gaining increasing relevance in recent

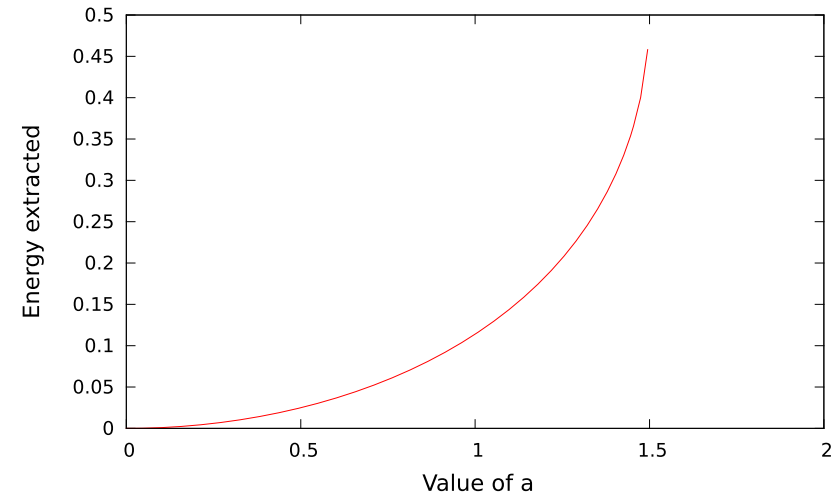

Fig. 2 Variation of energy extraction with axion for $M=2$ and $r_{2}=1$

times, explaining the production and nature of astrophysical jets that have originated from black holes. The most common process describing these is usually the Blandford and Znajek [16] mechanism or some allied models [17-19]. The main problem with such theories is that they are limited to charged particles and do not explain the neutral particles such as neutrons, neutrinos, photons, which are believed to be responsible for very long and thin gamma ray bursts. In this context, purely gravitational models were considered and the Penrose process of energy extraction was used to obtain a very energetic, perfectly collimated jet in a Kerr black hole spacetime without making use of magnetic fields [20]. It has been seen that there are two possible classes of geodesics in this spacetime. One with vertical asymptotes parallel to the $z$-axis which can form perfectly collimated jets and the other with horizontal asymptotes along the radial coordinate. The Penrose process takes place at the points of intersection of these geodesics inside the ergoregion, producing outgoing particles of greater energy than the energy of the particles that came in. These incoming particles could have originated from counter-rotating parts of the accretion disk surrounding the black hole, which gave rise to viscosity-induced instabilities which led to the infall of matter into the black hole. This model of the origin of the astrophysical jets as being via an energy extraction process from the black hole does not take recourse to the presence of charged particles and can explain the emission of neutral particles, such as very high energy neutrinos [21,22], as coming from the collimated inner jet. Massless particles such as photons [23-28] can be produced by charged particles accelerated along the collimated inner jet.

Thus the fact that the Penrose process is believed to be responsible for the emission of massive and massless neutral particles in astrophysical jets, along with our understanding of the Penrose process being a process of energy extraction from the axion field, opens up a new perspective to the process of astrophysical jet formation. It can be said now that the infalling matter from the accretion disk gains energy from the axion field and is ejected, to be observed as a collimated 
astrophysical jet. This is because the main body of results of this paper suggests that the axion field is responsible for the rotation of the Kerr axion dilaton black hole.The energy which the infalling, accreting matter gains can be said to be from the rotation of the black hole, or equivalently, from the axion field itself.The presence of these jets could be taken thus as evidence of the existence of the axion field.

\section{Conclusion}

The Newman-Janis prescription, going from the spherically symmetric charged dilaton coupled gravity solution to the axisymmetric charged axion-dilaton coupled solution [8], has been used to generate inequivalent black hole solutions. The axion in four dimensions is dual to the KalbRamond field strength, which can equivalently be interpreted as a space-time torsion and thus as an axisymmetric charged axion-dilaton metric, which resembles the Kerr metric. Hence it is a black hole solution in a string inspired torsioned space-time. We have explicitly constructed various components for the torsion from the axion field, which in turn is responsible for rendering angular momentum to the black hole. We therefore expect an energy extraction process in a manner similar to the well-known Penrose process. The presence of the dilaton also influences the space-time geometry and the energy extraction process. We show that at the end of the energy extraction, when the energy from the rotational energy of the black hole (or equivalently the energy of the axion or the Kalb-Ramond field) has been used up, we are left with a pure charged non-rotating dilaton black hole. This is, as already mentioned, a spherically symmetric geometry of spacetime, and hence we cannot expect any further energy to be extracted from the dilaton energy by means of the Penrose process.

As the black hole entropy is proportional to the area of its event horizon, we can define a quantity known as the irreducible mass for the axion-dilaton black hole proportional to the area of its outer event horizon. The change in this quantity for the axion-dilaton black hole can be shown to always be positive by defining the inner product of the four momentum of a test particle with a Killing vector which is actually a linear combination of the timelike and axial Killing vectors in the charged axion-dilaton black hole spacetime. When the energy of the axion is fully extracted, we are left with a pure charged dilaton black hole with a remnant entropy proportional to the area of the event horizon of the dilaton black hole. The difference between these two quantities measures the maximum amount of energy that can be extracted before the rotation of the black hole finally stops and the energy of the axion is fully extracted. The amount of energy extracted is plotted against the parameter measuring the dilaton and the axion field strength. The amount of energy extracted as well as the rate of extraction are found to decrease with the decrease in the dilaton and the axion field strength.

In this work, we have thus shown that, by an energy extraction process similar to the Penrose process, the geometry of the spacetime is being altered. The energy is being extracted from the axion/Kalb-Ramond field (or equivalently the rotational energy of the black hole). In the presence of the axion, we have an Einstein-Cartan-like spacetime, due to the equivalent description of the axion as a torsion of spacetime. When the energy of the axion is extracted fully, we are left with a charged pure dilaton black hole, described via torsionless geometry. This picture is, however, a matter of interpretation depending on whether one would like to interpret the KR field or the equivalent axion as an external tensor field or through the antisymmetric connection of space-time geometry. In light of current investigations regarding the origin of observed neutral emissions in astrophysical jets, a Penrose process acting on infalling matter from the surrounding accretion disk into the black hole, leading to the emission of highly energetic collimated jets, has been hypothesized [20]. In this regard, with reference to the main conclusion of this paper, it is possible to conclude that the energy of these neutral particle emissions is derived from the axion field, which is responsible for the rotational energy of the Kerr black hole. If this is so, then the origin of these astrophysical jets actually lies in the presence of the axion field as it derives its energy from it. This would perhaps shed new light on the little understood origin of these highly energetic cosmic jets.

Acknowledgments CG would like to thank the Inlaks Shivdasani Foundation and the Cambridge Nehru Bursary for funding during her studies in the University of Cambridge, where part of this work was done.

Open Access This article is distributed under the terms of the Creative Commons Attribution 4.0 International License (http://creativecomm ons.org/licenses/by/4.0/), which permits unrestricted use, distribution, and reproduction in any medium, provided you give appropriate credit to the original author(s) and the source, provide a link to the Creative Commons license, and indicate if changes were made.

Funded by SCOAP . $^{3}$

\section{References}

1. E. Newman, A. Janis, J. Math. Phys. 6, 915 (1965)

2. E. Newman, E. Couch, K. Chinnapared, A. Exton, A. Prakash, R. Torrence, J. Math. Phys. 6, 918 (1965)

3. S.F. Hassan, A. Sen (1991). arXiv:hep-th/9109038v1

4. A. Sen (1992). arXiv:hep-th/9204046v1

5. D. Garfinkle, G. Horowitz, A. Strominger, Phys. Rev. D 43, 3140 (1991)

6. D. Garfinkle, G. Horowitz, A. Strominger, Phys. Rev. D 45, 3888(E) (1992)

7. S. Yazadjiev (1999). arXiv:gr-qc/9906048

8. S. Yazadjiev (1999). arXiv:gr-qc/9907092v1

9. R.T. Hammond, Rep. Prog. Phys. 65, 599 (2002)

10. B. Mukhopadhyaya, S. SenGupta, Phys. Lett. B 458, 8 (1999) 
11. P. Majumdar, S. SenGupta, Class. Quantum Gravity 16, L89 (1999)

12. B. Mukhopadhyaya, S. SenGupta, S. Sur, Mod. Phys. Lett. A 17, $43(2002)$

13. B. Mukhopadhyaya, S. Sen, S. SenGupta, Phys. Rev. Lett. 89, 121101 (2002). [Erratum-ibid. 89, 259902 (2002)]

14. A. Sen (1991). arXiv:hep-th/9108011v1

15. S.M. Carroll, Lecture Notes on General Relativity (1997). arXiv:gr-qc/9712019v1

16. R.D. Blandford, R.L. Znajek, Mon. Not. R. Astron. Soc. 179, 433 (1977)

17. B. Punsly, Astrophys. J. 527, 609 (1999a)

18. B. Punsly, Astrophys. J. 527, 624 (1999b)

19. B. Punsly, Black Hole Gravitohydromagnetics (Springer, Berlin, 2001)

20. J. Gariel, M.A.H. MacCallum, G. Marcilhacy, N.O. Santos, Kerr geodesics, the Penrose process and jet collimation by a black hole (2010). arXiv:gr-qc/0702123v3

21. Auger Collaboration, Science 318, 939 (2007)
22. Auger Collaboration, Astropart. Phys. 29,188 (2007). [Erratum: ibid 30, 45 (2008)]. arXiv:0712.2843v2

23. C.D. Dermer, S. Razzaque, J.D. Finke, A. Atoyan (2008). arXiv:0811.1160v3

24. HESS Collaboration, Astrophys. J. Lett. (2009). arXiv:0903.1582v1

25. V.S. Semenov, S.A. Dyadechkin, M.F. Heyn, Buoyancy and Penrose process produce jets from rotating black holes (2014). arXiv: $1404.2474 \mathrm{v} 1$ [astro-ph.HE]

26. I.F. Mirabel, L.F. Rodriguez, Nature 371, 46 (1994)

27. I.F. Mirabel, L.F. Rodriguez, Ann. Rev. Astron. Astrophys. 37, 409 (1999)

28. I.F. Mirabel, Black holes: from stars to galaxies. Tech. Rep. (2006). arXiv:astro-ph/0612188. (Concluding Remarks of IAU Symposium 238: Black Holes: From Stars to Galaxies-Across the Range of Masses, Prague) 